ARTICLE

\title{
A transXend Detector - Principle and Applications
}

\author{
Ikuo KANNO* \\ Graduate School of Engineering, Kyoto University, Sakyo, Kyoto 606-8501, Japan
}

\begin{abstract}
A transXend detector was developed to use energy information of X-rays in computed tomography (CT). In this paper, the operation principle of the transXend detector is described first. The way of applying it to CT is following. Some possible fields to apply it are shown.
\end{abstract}

\section{KEYWORDS: computed tomography, energy information, unfolding, X-rays, contrast agent}

\section{Introduction}

In computed tomography (CT) measurements, contrast agent such as iodine is injected into blood vessel (contrast enhanced CT) to make a cancer tissue visible with the absorption of X-rays by iodine. The portion of blood vessel in the cancer tissue is higher than the one of normal tissue, and as a result, iodine concentration in the cancer tissue is higher than the one of normal tissue. The iodine-marked cancer tissue absorbs X-rays more than normal tissue and is observed easily.

The contrast enhanced CT, however, has two major shortcomings: the side effect of contrast agent"), and a "beam hardening effect"2). The beam hardening effect happens when a subject is thick, and an X-ray tube voltage is high: after passing a thick subject, the number of X-rays, which are sensitive to the absorption by iodine, becomes less. As a result, the X-ray absorption by iodine cannot be seen very clearly.

For avoiding these two disadvantages, the author employed the energy information of X-rays in CT measurement. The energy resolved CT measurements with using a CdZnTe detector demonstrated advantages over the conventional current measurement method in nearly twofold better iodine contrast, and insensitivity to the beam hardening effect ${ }^{3)}$. The measurement time, however, was very long: nearly 10 minutes for one $x$ - $\theta$ position of the first generation $\mathrm{CT}$ measurement.

In the X-ray CT, transmission measurement should be done quickly, if the subject is a human being. With this condition, the X-rays have been measured as electric current, i.e., the energy information of X-rays is discarded.

In the energy measurement of photons such as X-rays and gamma-rays, we have a limitation in counting rate in any type of radiation detectors. We have to wait for the charges, e.g., electrons and ions, electrons and holes, arrive at positive and negative electrodes, respectively.

* Corresponding author, E-mail: kanno@nucleng.kyoto-u.ac.jp,

(C) 2012 Atomic Energy Society of Japan, All Rights Reserved.
Most radiation detectors pursue the ability of measuring the energy of radiation as precise as possible. For high precision energy measurement of photons, photons should be collimated to limit the incident rate to the radiation detector. The energy measurement and high-flux photon measurement are not compatible.

In applications, the precise energy information, however, is not always necessary. Besides, it is not possible to measure the energy of high flux photons by conventional radiation detectors. Nevertheless, in some cases, even rough energy distribution of photons could bring useful information.

To make the energy resolved $\mathrm{CT}$ as a practical measurement method, the author developed a new detector, called a transXend detector. This transXend detector consists of several segment detectors, which are aligned along the direction of X-ray incidence, as shown in Figure 1. Each segment detector measures X-rays as electric current. Because the low energy X-rays are absorbed by the forward segment detectors and never reach to the rearward ones, and high energy X-rays can be absorbed by the rearward segment detectors, the energy distribution of incident X-rays is obtained by an unfolding method with using previously obtained response functions.

In this paper, a principle of the transXend detector is described first. A method of applying the transXend detector

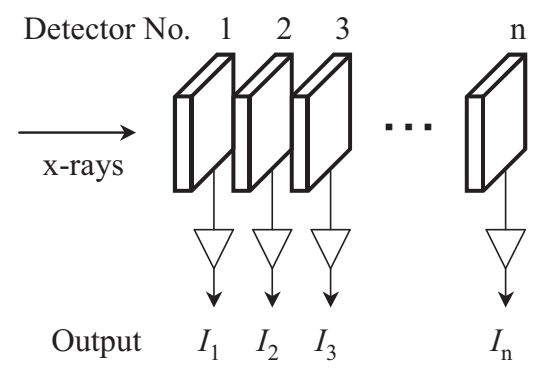

Fig. 1 Schematic drawing of the transXend detector. 
to the X-ray $\mathrm{CT}$ is explained in detail. After that, possible applications of transXend detectors will be mentioned.

\section{Principle of transXend detector}

When white X-rays come to a transXend detector, low energy X-rays are absorbed by the first segment detector. The energy spectrum of the incident $\mathrm{X}$-rays changes from the initial one when the X-rays come to the second segment detector. Although the substrates of the segment detectors are the same, the response functions of segment detectors differ to some extent due to the change of the energy spectrum of the X-rays incident to the detector. In case we have $n$ segment detectors and $n$ energy ranges to determine the number of X-rays in them, the relationship between the measured currents by the segment detectors, $I_{\mathrm{i}}(i=1, n)$, and the number of $\mathrm{X}$-rays $Y_{\mathrm{j}}(j=1, n)$, is written as,

$$
\left(\begin{array}{c}
I_{1} \\
I_{2} \\
\cdot \\
I_{n}
\end{array}\right)=\left(\begin{array}{cccc}
R_{11} & R_{12} & \cdot & R_{1 n} \\
R_{21} & & & \cdot \\
\cdot & & & \cdot \\
R_{n 1} & \cdot & \cdot & R_{n n}
\end{array}\right)\left(\begin{array}{c}
Y_{1} \\
Y_{2} \\
\cdot \\
Y_{n}
\end{array}\right) .
$$

Here, $R_{\mathrm{ij}}$ is the response function as a function of the segment detector position $i$ and the energy range $j$. With estimating the response function using known $\mathrm{X}$-ray absorbers, the energy distribution of X-rays is obtained by using an unfolding method.

The substrate of segment detectors can be chosen according to the X-ray energy of interest. The segment detector, especially forward segment detector, should not absorb all the X-rays. The substrate of a segment detector should have small atomic number and density, especially when it is placed as a forward segment detector.

In the case that the number of segment detectors and energy ranges are the same, the matrix in Eq.(1) is a regular matrix. A matrix equation with a regular matrix can be solved basically, however, sometimes the answer may not have physical meanings, i.e., negative X-ray events. For avoiding non-physical answers, utilization of an unfolding code such as SAND-II ${ }^{4)}$ is favorable.

\section{Application of transXend detector \\ 1.CT with $\mathrm{X}$-ray energy information}

(1) Experimental setup

The purpose of developing a transXend detector is the usage of energy information of X-rays in CT. Methods of estimating response functions and obtaining $\mathrm{CT}$ images are explained first.

Experimental setup for response function estimation is shown in Figure 2. An X-ray tube (TRIX 150S, Toreck Co., Ltd., Japan), $\mathrm{Pb}$ collimators, an acrylic phantom and a transXend detector are placed on the line. The employed $\mathrm{X}$-ray tube voltages are 80,100 and $120 \mathrm{kV}$. The $\mathrm{X}$-ray tube current is $2 \mathrm{~mA}$.

The acrylic phantom has the thickness of $7 \mathrm{~mm}$. It has four iodine regions with the thickness of $5 \mathrm{~mm}$ for thinned iodine tincture. The effective iodine thicknesses are 15, 30, 45 and $60 \mu \mathrm{m}$ in $5 \mathrm{~mm}$ water thickness. This acrylic phantom changes its position by a precision stage. Because the diameter of an acrylic phantom for CT measurements is $30 \mathrm{~mm}$, acrylic slabs with the thickness of $10 \mathrm{~mm}$ are incrementally added to this acrylic phantom with iodine regions to have the acrylic thickness 7-37 $\mathrm{mm}$.

In this experiment, $\mathrm{Si}(\mathrm{Li})$ detectors are employed for segment detectors. The dimensions of each $\mathrm{Si}(\mathrm{Li})$ detector is $10 \times 10 \times 1 \mathrm{~mm}^{3}$. These $\mathrm{Si}(\mathrm{Li})$ segment detectors are operated at room temperature without applying bias voltage as the mean free paths of electrons and holes are sufficiently long enough to arrive at each electrodes.

The current outputs from six segment detectors are amplified by a current preamplifier (IPA-6, Raytech Corp., Japan), and read out simultaneously by timer-counters with voltage-frequency converters (VFCT-8S4, Laboratory Equipments Corp., Japan). The position of the phantom and the timing of reading out segment detectors are controlled by a software, LabVIEW.

(2) Calculation of X-ray energy spectrum

The X-ray energy spectrum calculation is performed in the following way. The X-ray energy spectrum when emitted by the X-ray tube is written by Kramers formula ${ }^{5}$,

$$
\Psi_{0}(E) d E=\text { const. } \times Z \frac{E_{0}-E}{E} d E .
$$

Here, $E_{0}$ is the $\mathrm{X}$-ray tube voltage, $Z$ is the atomic number of X-ray tube target, and $E$ the energy of X-rays. The generated X-rays are attenuated by materials between the $\mathrm{X}$-ray tube and the detector. The energy spectrum of $\mathrm{X}$-rays when detected is,

$$
\Psi(E) d E=\Psi_{0}(E) \cdot \exp \left[-\sum_{x} \frac{\mu_{x}(E)}{\rho_{x}} \rho_{x} t_{x}\right] d E .
$$

Here, $\mu_{\mathrm{x}} / \rho_{\mathrm{x}}$ is the mass-attenuation coefficient, $\rho_{\mathrm{x}}$ is the density and $t_{\mathrm{x}}$ is the thickness of absorber material $x$. The number of X-rays in the energy range $j$ is obtained as,

$$
Y_{j}=\int_{E_{j}} \Psi(E) d E .
$$

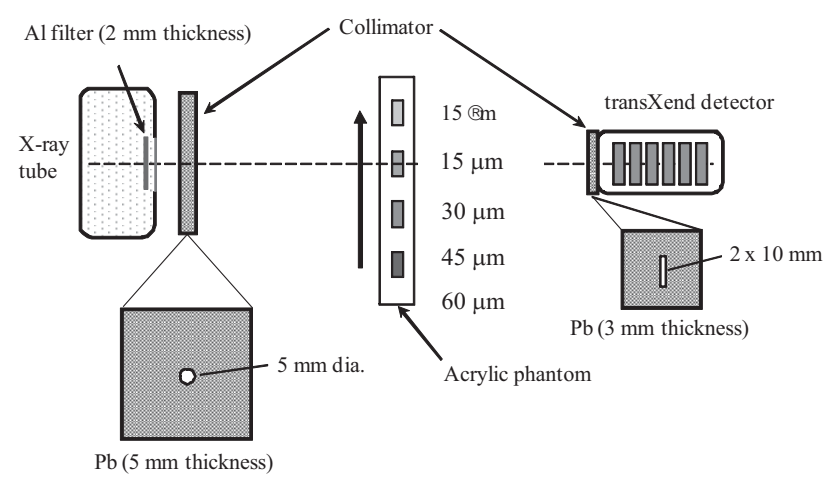

Fig. 2 Experimental setup. 
(3) Response function

Example of the measurement results of X-rays transmitted through the $7 \mathrm{~mm}$ thick rectangular acrylic phantom is shown in Figure 3 for the case of X-ray tube voltage $120 \mathrm{kV}$. In general, forward segment detector has greater current values than the rearward ones. However, in the data, the current values obtained by channel 5 are greater than the ones of channel 4 , because the gains of the preamplifiers are

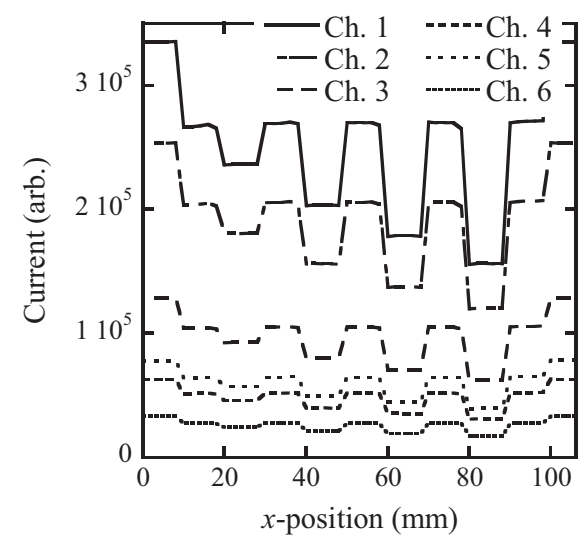

Fig. 3 Measured currents with scanning the square phantom with four iodine regions.

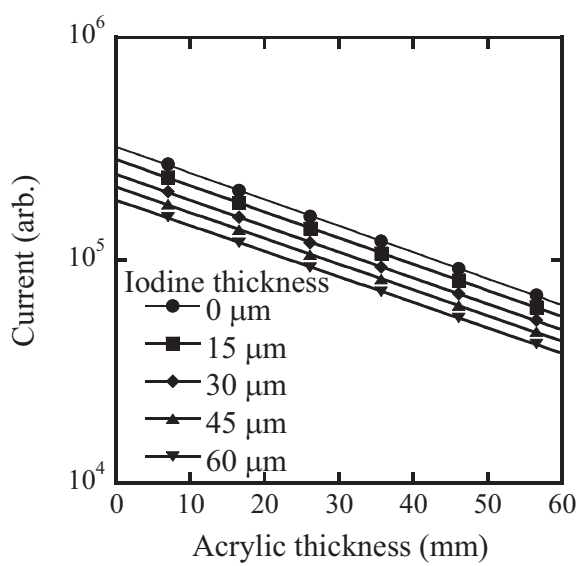

Fig. 4 Fitted current measured by the segment detector 1, as functions of acrylic and iodine thicknesses.

not the same. In Figure 4, fitted currents for the segment detector 1 are shown for X-ray tube voltage $120 \mathrm{kV}$ as functions of iodine and acrylic thickness.

When X-rays passed through the square phantom with the iodine thickness $d_{\mathrm{p}}(p=1, l)$, the $i$-th segment detector measures them as current $I_{\mathrm{i}}\left(d_{\mathrm{p}}\right)$. With the X-ray energy distribution $Y_{\mathrm{j}}\left(d_{\mathrm{p}}\right)$ in the energy range $j$ estimated by calculation, we have a following matrix equation,

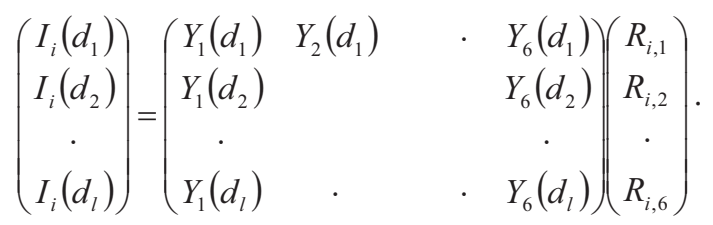

In the SAND-II code, the current for $i$-th segment detector after $k$-th iterative is,

$$
I_{i}^{(k)}\left(d_{p}\right)=\sum_{j=1}^{n} Y_{j}\left(d_{p}\right) R_{i, j}^{(k)}
$$

The weighting factor for iterative calculation is defined as,

$$
W_{i, j}^{(k)}\left(d_{p}\right)=\frac{Y_{j}\left(d_{p}\right) R_{i, j}^{(k)}}{I_{i}^{(k)}\left(d_{p}\right)} .
$$

The ratio of measured and $k$-th iterative currents is,

$$
r_{i}^{(k)}=I_{i} / I_{i}^{(k)} \text {. }
$$

The correction factor $C_{j}^{(k)}$ and the $(k+1)$-th response function $R_{i, j}^{(k+1)}$ are obtained in the followings, respectively.

$$
\begin{aligned}
& C_{i, j}^{(k)}=\frac{\sum_{p=1}^{l} W_{i, j}^{(k)}\left(d_{p}\right) \ln r_{i}^{(k)}\left(d_{p}\right)}{\sum_{p=1}^{l} W_{i, j}^{(k)}\left(d_{p}\right)} \\
& R_{i, j}^{(k+1)}=R_{i, j}^{(k)} \exp C_{i, j}^{(k)}
\end{aligned}
$$

The iteration calculation is terminated when the change of the current values between successive iteration is less than a conversion condition,

$$
v=\left|\frac{R_{i, j}^{(k+1)}}{R_{i, j}^{(k)}}-1\right| .
$$

We choose $v$ as $10^{-4}$ because the obtained results do not change significantly from the ones under the condition of $v=10^{-4}$, whereas calculation time increases enormously.

Table 1 Assigned energy ranges.

\begin{tabular}{ccccccc}
\hline \hline $\mathrm{keV}$ & $E_{1}$ & $E_{2}$ & $E_{3}$ & $E_{4}$ & $E_{5}$ & $E_{6}$ \\
\hline 80 & $15-25$ & $25-33$ & $33-40$ & $40-50$ & $50-60$ & $60-80$ \\
100 & $15-25$ & $25-33$ & $33-40$ & $40-60$ & $60-80$ & $80-100$ \\
120 & $15-25$ & $25-33$ & $33-40$ & $40-60$ & $60-90$ & $90-120$ \\
\hline
\end{tabular}

The response function should be obtained for the acrylic thickness from zero to that corresponding to the diameter of the cylindrical acrylic phantom for CT measurement. In our case, the response functions are estimated for $1 \mathrm{~mm}$ thick interval for acrylic.

Assigned energy ranges for response function estimation and CT image reconstruction are shown in Table $\mathbf{1 .}$

The obtained response functions for segment detectors are shown in Figure 5 for the X-ray tube voltage $120 \mathrm{kV}$.

\section{(4) Spectrum survey method}

The response functions obtained as Figure $\mathbf{5}$ do not differ much each other: the magnitude of the response function monotonously decreases as the channel number increases. The reason of the small change in the response functions is that the substrate of the segment detectors is the same. The change of response functions is a result of spectrum change due to the X-ray absorption by the forward segment detectors.

The unfolding process, which uses response functions similar to each other, does not provide definitive results. For quantitative evaluation of the results, a spectrum survey method is employed. 
With preparing X-ray energy distribution by calculation using Eq. (3) for acrylic and iodine thicknesses $1 \mathrm{~mm}$ and 5 $\mu \mathrm{m}$ intervals, the most probable X-ray energy distribution is chosen according to the following error estimation,

$$
\sigma=\sqrt{\frac{1}{n-1} \sum_{i=1}^{m}\left(\sum_{j=1}^{n} R_{i, j} Y_{j} / I_{i}-1\right)^{2}} .
$$

Here, $m$ and $n$ are the numbers of segment detectors and energy ranges, respectively. In Figure 6, the results of spectrum survey are shown for the iodine thicknesses of 0 , $15,30,45$ and $60 \mu \mathrm{m}$ with acrylic thickness $30 \mathrm{~mm}$. For every iodine thickness, the error is minimum when the initial guess spectra corresponding to each iodine thickness are employed.

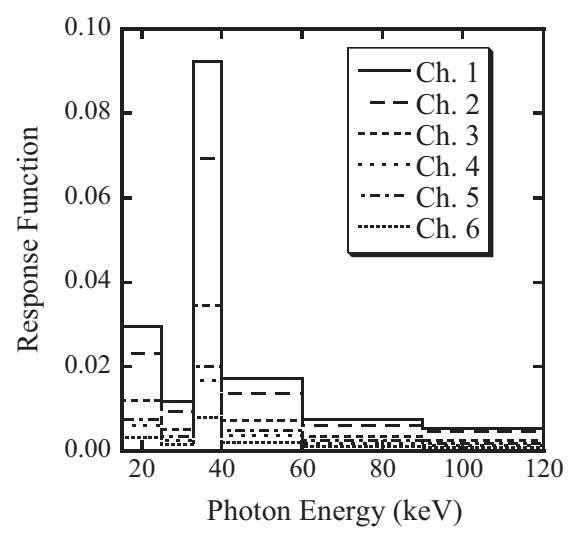

Fig. 5 Response functions. X-ray tube voltage is $120 \mathrm{kV}$.

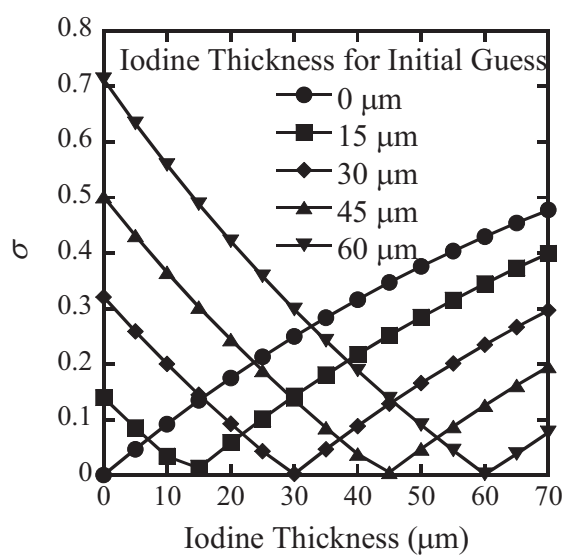

Fig. 6 Results of spectrum survey method.

\section{(5) CT measurements}

With replacing the square acrylic phantom by a cylindrical acrylic phantom, CT measurements are performed. The diameter of the cylindrical phantom is 30 $\mathrm{mm}$. The phantom has a $5 \mathrm{~mm}$ diameter hole in the center for thinned iodine tincture. The effective thickness of the iodine is $15 \mu \mathrm{m}$ in $5 \mathrm{~mm}$ thick water. The CT measurements are carried out with translating the phantom with $0.4 \mathrm{~mm}$ step, and 10 degrees rotations. In practice, the rotation angle is changed for 60 degrees. With duplicating the rotation data for 5 times, we have 10 degree interval rotation data. The current data obtained by the scanning

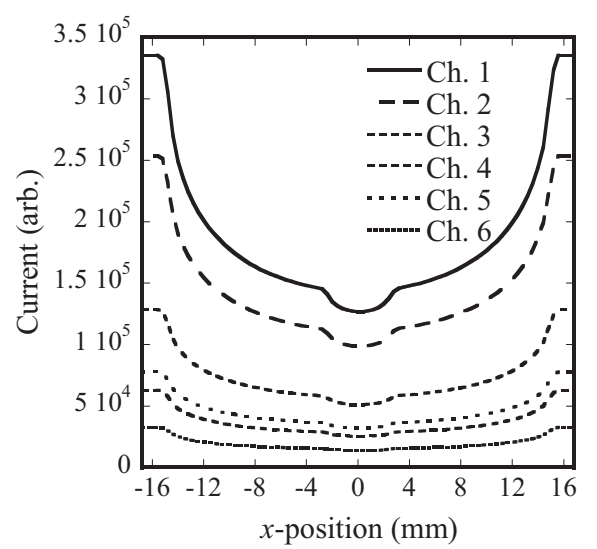

Fig. 7 Scanned current data for tube voltage $120 \mathrm{kV}$.

with each segment detector are shown in Figure 7 for 120 $\mathrm{kV}$ tube voltage.

With adding the current data of all the segment detectors, conventional current data is obtained. A CT image is reconstructed by the current data first. With this CT image, the X-ray path length at each measurement point is estimated. With the current data and the X-ray path length, iodine thickness is estimated for each energy range. Estimated acrylic and iodine thickness in a scanned data is shown in Figure 8 for the case of X-ray tube voltage $120 \mathrm{kV}$.

For applying the spectrum survey method, initial guess energy distributions are prepared for iodine thickness with 1 $\mu \mathrm{m}$ intervals. The acrylic thickness is fixed to the X-ray path length of the data point. With using SAND-II and Eq. (12), an initial guess which gives the minimum error is chosen as an answer.

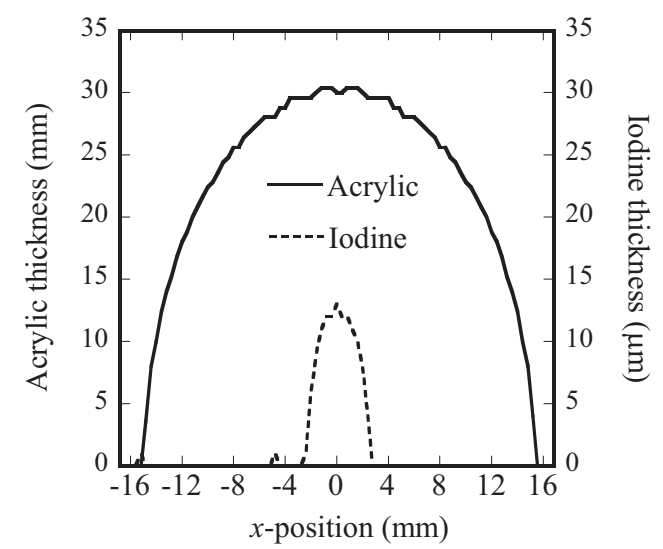

Fig. 8 Estimated acrylic and iodine thickness as a function of phantom position.

In Figure 9, CT images reconstructed from the obtained X-ray events in each energy range are shown. The reconstruction program is the one using the algorism of maximum likelihood expectation maximization method ${ }^{6}$. The difference between the CT values of acrylic and iodine regions is the maximum in the energy range $E_{3}$, i.e., energy range higher than the $\mathrm{K}$-edge of iodine. The $\mathrm{CT}$ value profiles obtained by the X-ray events for $E_{3}$ along the diameter of the phantom are shown in Figure 10 for X-ray tube voltages 80,100 , and $120 \mathrm{kV}$, as well as the ones obtained by current measurement method. The CT values obtained by current measurement become smaller in the 


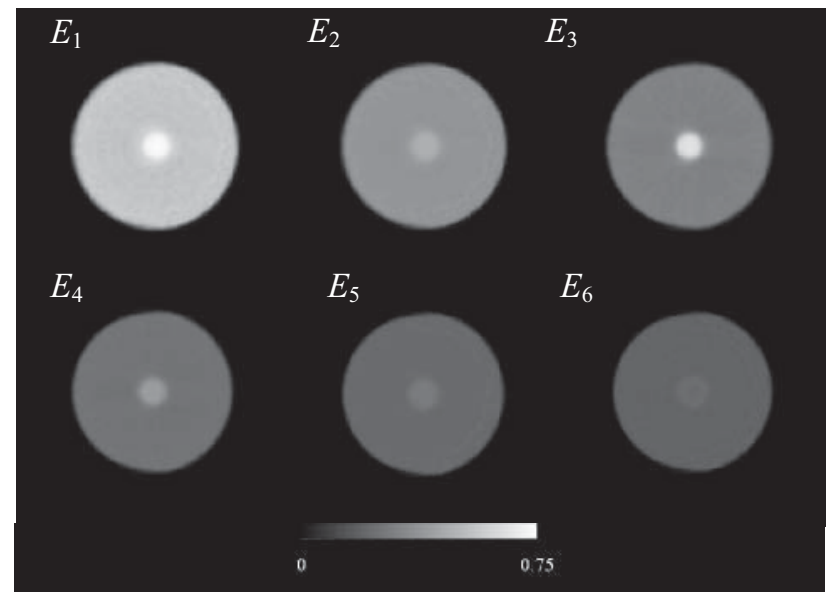

Fig. 9 CT images obtained by X-rays in each energy range.

higher X-ray tube voltage, but, the energy resolved CT values stay the same irrespective of the tube voltages.

\section{Gamma ray measurement of mixed isotopes}

(1) CT measurements with mixed gamma-rays

With a CT measurement using two monochromatic $\mathrm{X}$-rays, the atomic number and electron density distributions can be estimated. ${ }^{7)}$ For this kind of measurements, a CT measurement with a monochromatic X-rays should be repeated with changing the energy of X-rays, if the X-rays are measured as electric current.

If the transXend detector is employed, the number of CT measurements can be reduced to once, when a subject is exposed to dual monochromatic X-rays, simultaneously.

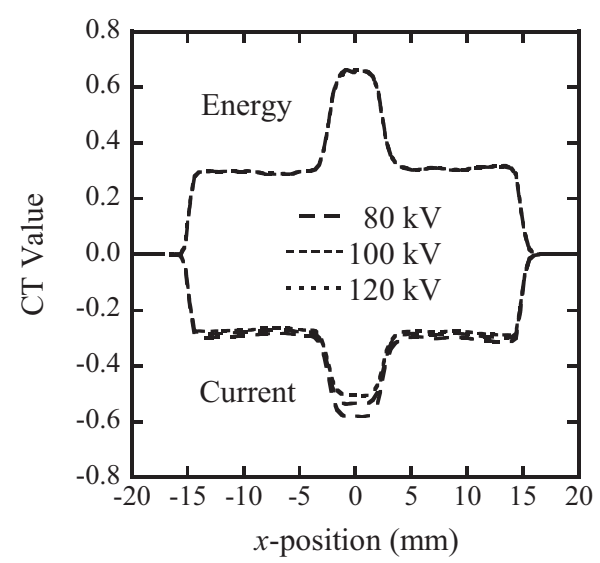

Fig. 10 CT value profiles for energy information and conventional current CT. Current CT profiles are plotted in negative values for clear comparison.

The possibility of distinguishing two kinds of gamma rays for ${ }^{137} \mathrm{Cs}$ and ${ }^{60} \mathrm{Co}$ is discussed using a simulation calculation with the convergence condition of $10^{-4}$ : the unfolding in the mixed field of ${ }^{137} \mathrm{Cs}$ and ${ }^{60} \mathrm{Co}$ may be difficult if the fraction of ${ }^{60} \mathrm{Co}$ is too high because the gamma ray energy of ${ }^{137} \mathrm{Cs}$ is nearly one half of that of ${ }^{60} \mathrm{Co}^{8}{ }^{8)}$ The substrate of segment detectors for simulation calculation is CdTe with the thickness of $10 \mathrm{~mm}$. As shown in Figure 11, ${ }^{137} \mathrm{Cs}$ and ${ }^{60} \mathrm{Co}$ gamma rays can be distinguished by the unfolding if the number of ${ }^{137} \mathrm{Cs}$ gamma rays is greater than $10 \%$ and less than 300 times of that of ${ }^{60} \mathrm{Co}$ gamma rays.

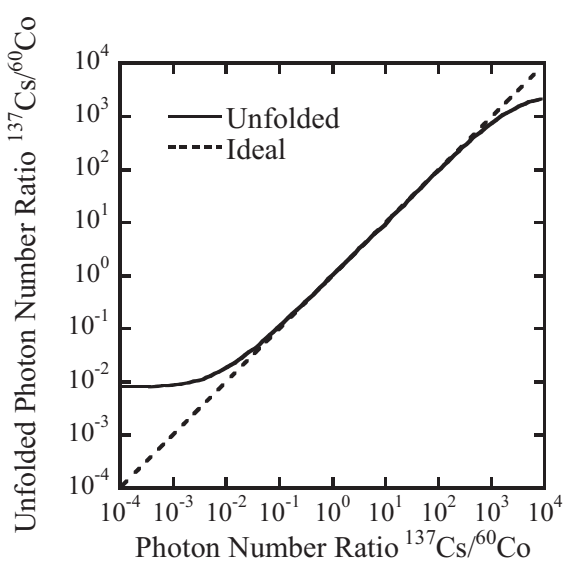

Fig. 11 Simulated possibility of unfolding ${ }^{137} \mathrm{Cs}$ and ${ }^{60} \mathrm{Co}$ gamma rays in mixed field as a function of ratio of the number of gamma rays from each source.

CT measurements are performed for an iron phantom with a diameter of $30 \mathrm{~mm}$ with using ${ }^{137} \mathrm{Cs}(4.44 \mathrm{TBq})$ and ${ }^{60} \mathrm{Co}$ (37 GBq) sources, alternatively. The iron phantom has an aluminum and a brass rods with $10 \mathrm{~mm}$ diameters. The measurements are carried out with $1 \mathrm{~mm}$ step translation and 10 degrees rotation with a transXend detector of three CdTe with $10 \mathrm{~mm}$ thick, $5 \mathrm{~mm}$ wide and $5 \mathrm{~mm}$ high. Current data obtained for each radioisotope are added and resulted in the data for mixed radioisotopes. After unfolding the mixed data with initial guess spectrum consisting of $0.662,1.17$ and $1.33 \mathrm{MeV}$ gamma rays, CT images are obtained for Cs gamma rays of $0.662 \mathrm{MeV}$ and $\mathrm{Co}$ gamma rays of $1.17,1.33$ $\mathrm{MeV}$ in energy. CT values along the diameter passing through aluminum, brass and iron in the phantom are shown in Figure 12.

(2) Estimation of $Z$ and $\rho_{\mathrm{e}}$ distribution

The mass absorption coefficient at energy $E, \mu(E)$ is written as, ${ }^{7)}$

$$
\mu(E)=\rho \frac{N_{A}}{A}\left\{\sigma_{\text {photo }}(Z, E)+\sigma_{\text {incoh }}(Z, E)+\sigma_{\text {pair }}(Z, E)\right\} .
$$

Here, $\rho, N_{\mathrm{A}}$ and $A$ are the density of the material, Avogadro's number, and atomic mass, respectively. $\sigma_{\text {photo, }}$ $\sigma_{\text {incoh }}, \sigma_{\text {pair }}$ are the cross sections of photoelectric absorption, incoherent scattering, and pair creation. With changing the energy of monochromatic X-rays, the ratio of mass absorption coefficient is obtained as a function of $Z$.

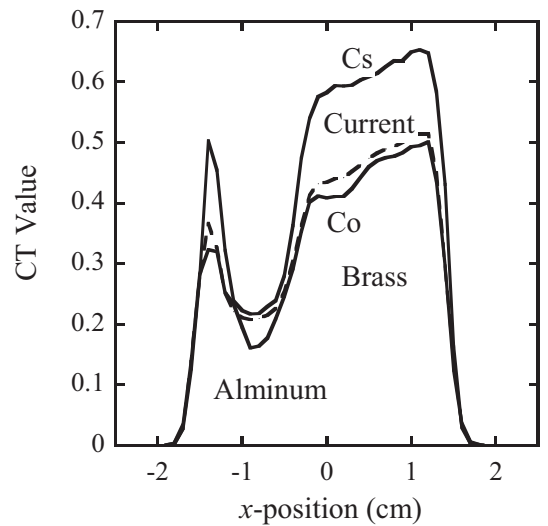

Fig. $12 \mathrm{CT}$ value profiles obtained by ${ }^{137} \mathrm{Cs}$ and ${ }^{60} \mathrm{Co}$ gamma rays. 
From the CT images, the averaged $\mu_{\mathrm{Cs}}$ and $\mu_{\mathrm{Co}}$ are estimated for aluminum, brass and iron. With the previously obtained relationship between $\mu_{\mathrm{Cs}} / \mu_{\mathrm{Co}}$ and $Z$, as shown in Figure 13, the atomic number is estimated.

The estimated atomic numbers and electron densities for three materials are shown in Table 2 , as well as the reference values. Although the reference values of iron are within the errors of estimated values, the results for aluminum and brass are slightly out of the error range. This not very successful results are due to the fact that the energies of gamma-rays are very higher than the energy range where the absorption cross sections of materials are effective, i.e., under $100 \mathrm{keV}$.

Table 2 Measured attenuation coefficients and estimated atomic numbers of iron, brass and aluminum. 'Meas.' shows the estimated values. 'Ref.' means the atomic numbers of elements.

\begin{tabular}{|c|c|c|c|}
\hline & Iron & Brass & Aluminum \\
\hline$\mu_{\mathrm{Cs}}\left(10^{-1} \mathrm{~cm}^{-1}\right)$ & $5.76 \pm 0.02$ & $5.92 \pm 0.01$ & $2.24 \pm 0.02$ \\
\hline$\mu_{\mathrm{Co}}\left(10^{-1} \mathrm{~cm}^{-1}\right)$ & $4.21 \pm 0.02$ & $4.23 \pm 0.03$ & $1.64 \pm 0.05$ \\
\hline$\mu_{\mathrm{Cs}} / \mu_{\mathrm{Co}}$ & $1.37 \pm 0.008$ & $1.40 \pm 0.01$ & $1.37 \pm 0.04$ \\
\hline$Z \quad$ Meas. & $19.0-27.9$ & $32.5-37.2$ & $0-37$ \\
\hline Ref. & 26 & 29,30 & 13 \\
\hline
\end{tabular}

\section{Transmission measurements}

The applications described above are on the CT measurements. The transXend detectors are also useful in simple transmission measurements such as chest radiography, and in interventional radiology. A method of applying transXend detectors to baggage inspection will be developed.

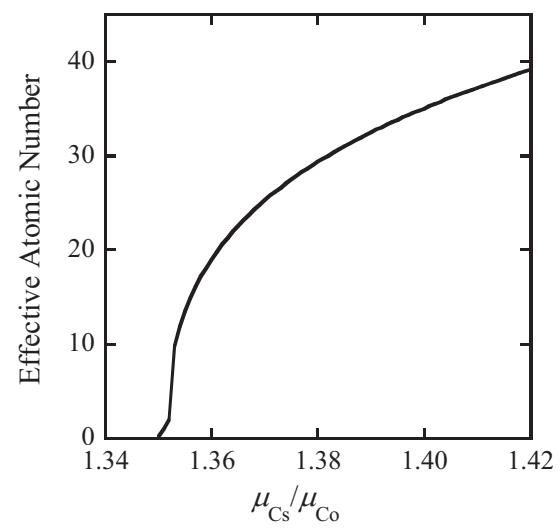

Fig. 13 Relationship between the photon absorption coefficient ratio and the effective atomic number.

\section{Conclusion}

For obtaining energy information of high flux photons, such as X-rays emitted by an X-ray tube for CT measurements, an idea of a transXend detector is introduced and the operating principle is described in the case of applying it in CT. The transXend detector is useful in identifying the atomic numbers in a subject, if dual monochromatic X-rays are available in the energy range below $100 \mathrm{keV}$. Besides, the number of exposure is reduced to only once, compared with the conventional current measurement method.

\section{Acknowledgements}

This paper is the review of our activity on transXend detectors. The author is very thankful to his collaborators: Mr. R. Imamura, Ms. Y. Minami, Mr. M. Ohtaka, Dr. M. Hashimoto, Dr. K. Ara, and Mr. H. Onabe. Also, he is deeply grateful to Prof. K. Watanabe, Nagoya University, for allowing the use of his CT image reconstruction program. This work was supported by the Suzuken Memorial Foundation.

\section{References}

1) H. Katayama, K. Yamaguchi, T. Kozuka, T. Takashima, P. Seez, K. Matsuura, "Adverse reactions to ionic and nonionic contrast media. A report from the Japanese committee on the safety of contrast media", Radiology, 175, 621-628 (1990).

2) R. A. Brooks, G. Di Chiro, "Beam hardening in X-ray reconstructive tomography", Phys. Med. Biol., 21, 390-398 (1976).

3) I. Kanno, A. Uesaka, S. Nomiya, H. Onabe, "Energy measurement of X-rays in computed tomography for detecting contrast media," J. Nucl. Sci. Technol., 45, 15-24 (2008).

4) W. McElroy, S. Berg, T. Crocket, G. Hawkins, $A$ computer-automated iterative method for neutron flux spectra determination by foil activation, AFWL-TR-67-41 (1967).

5) E. Storm, "Calculated bremsstrahlung spectra from thick tungsten targets", Phys. Rev., A5, 2328-2338 (1972).

6) P. P. Bruyant, J. Sau, J. J. Mallet, "Streak artifact reduction in filtered backprojection using a level line-based interpolation method," J. Nucl. Med., 41, 1913-1919 (2000).

7) R. A. Rutherford, B. R. Pullan and I. Isherwood, "Measurement of effective aomic number and electron density using an EMI scanner", Neuroradiology 11, 15-21 (1976).

8) M. Hashimoto, M. Ohtaka, K. Ara, I. Kanno, R. Imamura, K. Mikami, S. Nomiya and H. Onabe, "Simulation study on unfolding methods for diagnostic X-rays and mixed gamma rays", J. Nucl. Sci. Technol., 46, 76-82 (2009). 